Mathematische Semesterberichte manuscript No.

(will be inserted by the editor)

Jesús A. De Loera

\title{
The Many Aspects of Counting Lattice Points in Polytopes *
}

\begin{abstract}
A wide variety of topics in pure and applied mathematics involve the problem of counting the number of lattice points inside a convex bounded polyhedron, for short called a polytope. Applications range from the very pure (number theory, toric Hilbert functions, Kostant's partition function in representation theory) to the most applied (cryptography, integer programming, contingency tables). This paper is a survey of this problem and its applications. We review the basic structure theorems about this type of counting problem. Perhaps the most famous special case is the theory of Ehrhart polynomials, introduced in the 1960s by Eugène Ehrhart. These polynomials count the number of lattice points in the different integral dilations of an integral convex polytope. We discuss recent algorithmic solutions to this problem and conclude with a look at what happens when trying to count lattice points in more complicated regions of space.
\end{abstract}

\section{Introduction}

The main actors of this story are convex polytopes (e.g. cubes, triangles, and their high dimensional analogues) and the points with integer coordinates that lie inside them, i.e. their lattice points. The convex polytopes we care about here will most likely be encoded in a computer, and for this reason they will be represented as the sets of non-negative solutions of a system of linear equations $A x=b$, for suitable integral matrix $A$ and vector $b$. This is not at all a restrictive formulation; any system of linear inequalities and equalities with rational coefficients can be put into this form (the books $[7$, $53,60]$ are good references for many concepts used here). We will start now setting up our mathematical tale:

Given a polytope $P=\{x: A x=b, x \geq 0\}$, specified by $d \times n$ integral matrix $A$ and an integral $d$-vector $b$, i.e. $P=\{x: A x=b, x \geq 0\}$, our goal

\footnotetext{
* The author gratefully acknowledges the financial support received from NSF grant DMS-0309694, an Alexander von Humboldt fellowship, and the UC Davis Chancellor's fellow Award.

Jesús A. De Loera: Department of Mathematics, University of California, Davis CA 95616-8633, U.S.A. email: deloera@math.ucdavis.edu
} 
is to count how many lattice points are inside P. We are in fact interested on the counting function

$$
\phi_{A}(b)=\#\{x: A x=b, x \geq 0, x \text { integral }\} .
$$

This is the function that counts the number of lattice points inside convex polytopes given in terms of a fixed matrix $A$ and a right-hand-side vector $b$ that is possibly changing or consists of variables. In the first part of this survey I hope to convince you that the functions $\phi_{A}$ are very useful and natural. As we will see applied mathematicians often work with fixed $b$ and would perhaps prefer fast evaluation of $\phi_{A}(b)$ for fixed values of $b$. Pure mathematicians, on the other hand, would perhaps prefer to compute explicit exact formulas in terms of variables $b_{i}$.

Take the following example. If $A=[3,5,17]$ then we have a plane intersecting the nonnegative orthant, as presented in Figure 1, the polytope $P$ is a triangle (embedded in three-dimensional space). It gives us a function $\phi_{A}(n)=\#\{(x, y, z): 3 x+5 y+17 z=n, x \geq 0, y \geq 0, z \geq 0\}$. Thus, for example, $\phi_{A}(58)=9, \phi_{A}(101)=25, \phi_{A}(1110)=2471$, etc. For us a fine general formula for $\phi_{A}(b)$ could be the generating function whose terms encode the different values of $\phi_{A}$ :

$$
\sum_{n=0}^{\infty} \phi_{A}(n) t^{n}=\frac{1}{\left(1-t^{17}\right)\left(1-t^{5}\right)\left(1-t^{3}\right)}
$$

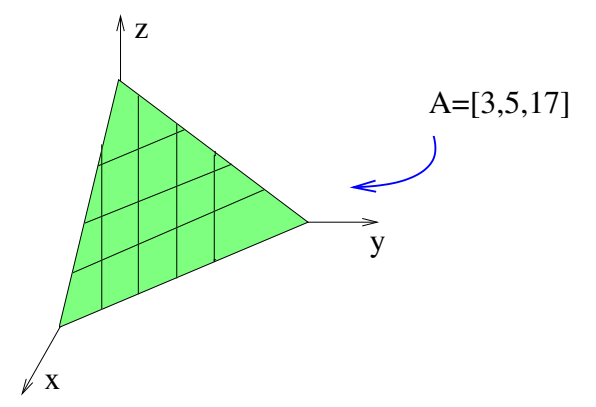

Fig. 1. Lattice points on a spatial triangle.

In the second part of the survey, I will outline the structure theory of the counting functions $\phi_{A}(b)$ and some useful algorithms to compute $\phi_{A}(b)$ involving rational functions. By the way, whenever I say counting, I mean exact counting. There is a rich and exciting theory of estimation and 
approximation, but that is a different story (see for example $[27,38,59]$ ). Now we present

\section{Applications}

\section{1. in Applied Mathematics:}

- Let us begin with two puzzles about coins and cars. A curious cashier may wonder: How many ways are there to give change of 10 dollars into pennies ( $p)$, nickels ( $n)$, dimes (d), quarters ( $q$ ), using exactly 100 coins? The answer is equivalent to the number of solutions of the system of linear equations

$$
p+5 n+10 d+25 q=10000, \quad p+n+d+q=100,
$$

where $p, n, d, q$ are nonnegative integral numbers. This is a reasonable exercise, but do not be fooled into thinking that problems with few constraints are always easier than those with many equations. Even with just one single row for the matrix $A$ there are hard instances that cannot be solved so by mere brute force enumeration (i.e. trying to list all possibilities). For instance, from the article [1], a very hard instance is $\left\{(x, y, z, w, v) \in \mathbb{R}_{\geq 0}^{5}: 12223 x+12224 y+36674 z+61119 w+85569 v=\right.$ $89643481\}$.

We can imagine $x, y, x, w, v$ are the five (integral) quantities of cars or trucks, from five different models, with weights (in kilograms) 12223, 12224 , etc. and we are supposed to completely fill in a ferry boat with maximal capacity of 89643481 kilograms. How many ways are there to do this? This is like filling a sack with objects up to the maximum capacity; this is why problems with a single-row matrix receive the name of knapsack problems. Knapsack problems are geometrically the simplest possible since their polytopes are simplices, the generalization of triangles for higher dimensions. Note that the counting problem can easily be turned into an optimization problem if we just ask: How can we fit the most cars?

- Continuing with optimization problems. Suppose you have a computer network between Berlin and Magdeburg. You are interested to send as many email messages as possible given its layout. In Figure 2 (A), Berlin and Magdeburg are represented as nodes 1 and 6 respectively. The other four nodes represent nearby cities within the network. Every time we send an e-mail, if it enters a node, it has to leave that node (conditions $b_{i}=0$ means no loss of information). There is also some capacity associated to each connecting phone line or internet cable that limits the number of messages we can send (e.g. between node 2 and 4 there is a capacity $c_{24}$ of 5 messages). 


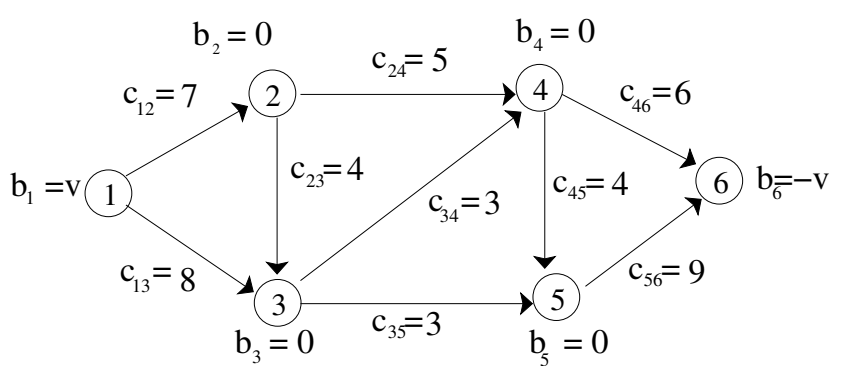

Fig. 2. A simple network.
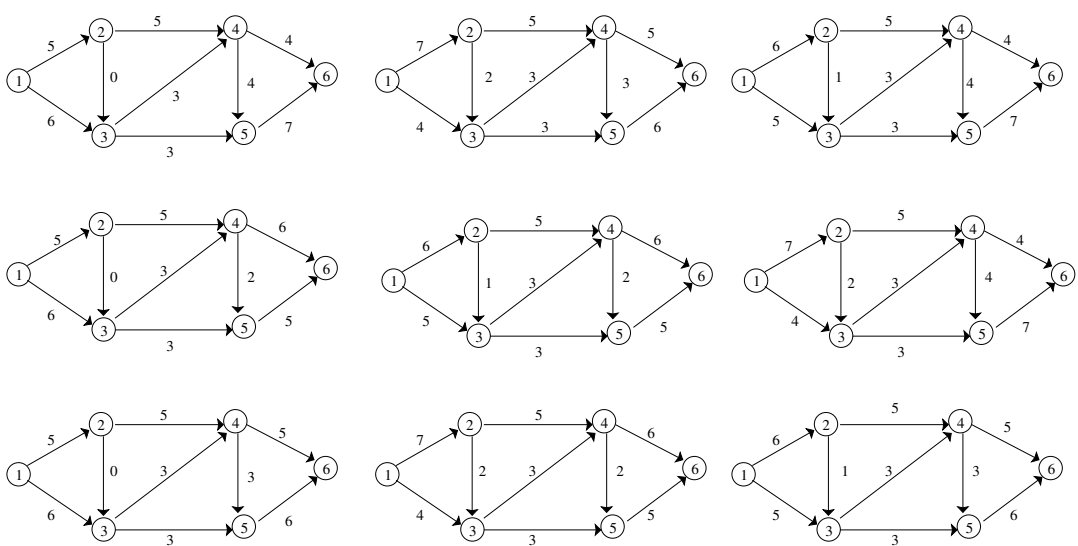

Fig. 3. All integral maximum flows in the network.

Classical and widely-known theorems [53] indicate that the largest number of email messages one can send for this example is 11. But how many distinct optimal solutions are there? It turns out that there are exactly 9 ways to route 11 emails in this small network (see Figure 3 (B)). In general, if $G$ is a network with $n$ nodes and $m$ arcs, with integer-valued capacities for the arcs and excess function for the nodes (namely $c: \operatorname{arcs}(G) \rightarrow \mathbf{Z}_{\geq 0}$ and $b: \operatorname{nodes}(G) \rightarrow \mathbf{Z}$ ). A flow is a function $f: \operatorname{arcs}(G) \rightarrow \mathbf{Z}_{\geq \mathbf{0}}$ so that, for any node $x$, the sum of flow values in outgoing arcs minus the sum of values in incoming arcs equals $b(x)$, and $0 \leq f(i, j) \leq c(i, j)$. We may be interested on knowing all maximal integral-valued flows or at least many of them. This is equivalent to counting the number of lattice points of a flow polytope (see [6] and references therein). This may be relevant for the security of communication networks, if we have only one or a few optimal solutions, this may indicate some kind of weakness in case of an attack [5].

- Suppose you are a compiler designer, then you would typically worry about how often is a certain instruction I of the computer code executed? Take for instance the following toy example (this program has really no 
particular meaning, it simply repeats instruction $I$ a few times depending on values of $N$ and $M$ ):

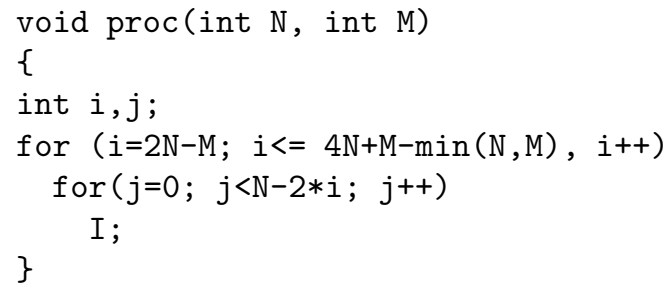

Clearly, the number of times we reach instruction $I$ would depend on $\mathrm{N}, \mathrm{M}$. In terms of these parameters the set of all possible solutions is given by the number of lattice points inside of parametric polygons. In our toy example these are described by the linear conditions

$\left\{(i, j) \in \mathbb{Z}^{2}: i \geq 2 N-M, i \leq 4 N+M-\min (N, M), j \geq 0, j-2 i \leq N-1\right\}$.

The answers to many questions about the computer performance or resource allocation available while compiling code with nested loops depends on counting lattice points of polytopes. Examples include how many memory locations are touched during a loop? How many cache misses does a loop generate? How much memory is dynamically allocated by a portion of code? See more details and references in [20,33, 45].

- Statistics is a topic where integral data tables are studied and generated a plenty. Take for instance the following 3-dimensional table of format $2 \times 3 \times 3$ given below. Table 1 has been extracted from the 1990 decennial USA census. Visit the web page of the U.S. census http://factfinder . census.gov/ where you can extract your own contingency tables! There are a variety of statistical questions where one may consider the "totals margins" (e.g. total number of white male respondents was 329, the number of chinese females is one, etc.) as fixed and we may wish to study the space of all integral tables that satisfy these sum restrictions on their entries. For example, counting those tables is closely related to the problem of sampling such a table uniformly at random or with respect to other probability distribution model. Another application is when we want to decide whether two of the traits of the statistic, say, income and race, are independent of each other. Several tests of independence depend on counting the integral tables in question. See [25, 30] and its references. The spaces of tables with fixed marginal totals are again polytopes, the so called transportation polytopes. The lattice points inside transportation polytopes receive the name of contingency tables. For example, if we consider our census table above, there are 18 entries possible depending on gender $(f, m)$, race $(w, b, c)$, and income 
Gender $=$ Male

Income Level

\begin{tabular}{|l|c|c|c||c|}
\cline { 2 - 5 } \multicolumn{1}{c|}{ Race } & $\leq \$ 10,000$ & $>\$ 10000$ and $\leq \$ 25000$ & $>\$ 25000$ & Total \\
\hline White & 96 & 72 & 161 & 329 \\
\hline Black & 10 & 7 & 6 & 23 \\
\hline Chinese & 1 & 1 & 2 & 4 \\
\hline \hline Total & 107 & 80 & 169 & 356 \\
\hline
\end{tabular}

Gender $=$ Female

Income Level

\begin{tabular}{|l|c|c|c||c|}
\cline { 2 - 5 } \multicolumn{1}{c|}{ Race } & $\leq \$ 10,000$ & $>\$ 10000$ and $\leq \$ 25000$ & $>\$ 25000$ & Total \\
\hline White & 186 & 127 & 51 & 364 \\
\hline Black & 11 & 7 & 3 & 21 \\
\hline Chinese & 0 & 1 & 0 & 1 \\
\hline \hline Total & 197 & 135 & 54 & 386 \\
\hline
\end{tabular}

Table 1. Three-way cross-classification of Gender, Race, and Income for a selected U.S. census tract. Source: 1990 Census Public Use Microdata Files.

values $(1,2$ or 3$)$. Since we fixed the "line" sums, we have, for example, a linear equation

$$
x_{m, w, 1}+x_{m, w, 2}+x_{m, w, 3}=329
$$

that explains that the number of white males is 329 when we add the three categories of income. There is a similar equation $x_{m, b, 2}+x_{f, b, 2}=$ 14 that indicates the number of blacks (female and male) with middle income must be 14. Altogether we will have 21 linear equations and 18 non-negative variables (one for each combination of possible gender, race, income) describing the polytope. How many integral points does it contain?

\section{2. in Pure Mathematics}

- Many combinatorial structures can be counted as lattice points of polytopes. For example, matchings on graphs [46], t-designs [51], linear extensions of posets [56], and of course magic squares! Magic squares are square arrays of nonnegative integer numbers where the sums along each row, column, or diagonal is a constant, the so called magic sum. In Figure 4 we see an example of a $4 \times 4$ magic square with magic sum 24 .

How many $4 \times 4$ magic squares with sum s are there? Denote by $f_{4 \times 4}(s)$ the number of $4 \times 4$ magic squares with magic sum $s$. Using the latest methods for counting lattice points we can present a nice formula for $f_{4 \times 4}(s)$ (and other magic objects [3]): 


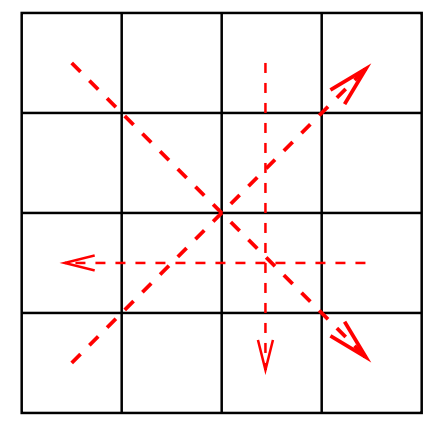

\begin{tabular}{|c|c|c|c|}
\hline 12 & 0 & 5 & 7 \\
\hline 0 & 12 & 7 & 5 \\
\hline 7 & 5 & 0 & 12 \\
\hline 5 & 7 & 12 & 0 \\
\hline
\end{tabular}

Fig. 4. A $4 \times 4$ magic square.

$$
\left\{\begin{array}{l}
\frac{1}{480} s^{7}+\frac{7}{240} s^{6}+\frac{89}{480} s^{5}+\frac{11}{16} s^{4}+\frac{49}{30} s^{3}+\frac{38}{15} s^{2}+\frac{71}{30} s+1 \\
\frac{1}{480} s^{7}+\frac{7}{240} s^{6}+\frac{89}{480} s^{5}+\frac{11}{16} s^{4}+\frac{779}{480} s^{3}+\frac{593}{240} s^{2}+\frac{1051}{480} s+\frac{13}{16} \text { if } 2 \not s .
\end{array}\right.
$$

In other words $f_{4 \times 4}(1)=8, f_{4 \times 4}(2)=48, f_{4 \times 4}(3)=200, f_{4 \times 4}(4)=$ $675, f_{4 \times 4}(5)=1904$, etc. All values can be read from the two-case formula, which is applied according with the parity of the magic sum. This formula, given in finitely many polynomial pieces, is a good example of a quasipolynomial [57]. We will come back to this later. The important thing to notice right now is that $f_{4 \times 4}(s)$ is again one of our functions $\phi_{A}(b)$ for a polytope which is almost a transportation polytope: The matrix $A$ is read off from the row, column, and diagonal sums for the entries of a $4 \times 4$ magic array. Our polytope lives in sixteen dimensional space, and $b$ is just $s(1,1, \ldots, 1)$.

- Researchers have observed that polytopes play a special role in representation theory (e.g. $[16,21,41,42])$. One example of such polytopes comes from the Gelfand-Tsetlin patterns which arise in the representation theory of $\mathfrak{g l}_{n} \mathbb{C}$. What is a Gelfand Tsetlin pattern? It is a triangular array of numbers $\mathbf{x}=\left(x_{i j}\right)_{1 \leq i \leq j \leq n}$ satisfying that $x_{i j} \geq 0$, for $1 \leq i \leq j \leq n$; and $x_{i, j+1} \geq x_{i j} \geq x_{i+1, j+1}$, for $1 \leq i \leq j \leq n-1 . x_{i n}=\lambda_{i}$ if $1 \leq i \leq n$, for $\lambda \in \mathbb{R}^{n}$. Given $\lambda, \mu \in Z^{n}$, the Gelfand-Tsetlin polytope $G T(\lambda, \mu) \subset X_{n}$ is the convex polytope of GT-patterns $\left(x_{i j}\right)_{1 \leq i \leq j \leq n}$ satisfying in addition that $x_{11}=\mu_{1}$; and $\sum_{i=1}^{j} x_{i j}-\sum_{i=1}^{j-1} x_{i, j-1}=\mu_{j}$, for $2 \leq j \leq n$.

The importance of GT-polytopes stems from a classic result of I. M. Gelfand and M. L. Tsetlin, which states that the number of integral lattice points in the Gelfand-Tsetlin polytope $G T(\lambda, \mu)$ equals the dimension of the weight $\mu$ subspace of the irreducible representation of $\mathfrak{g l}_{n} C$ with highest weight $\lambda$. If you are not familiar with representation theory let me also say that the lattice points of Gelfand-Tsetlin polytopes also have a nice combinatorial interpretation as semi-standard tableaux 
of of shape $\lambda$ and content $\mu$. The number of such tableaux is a Kostka number.

\begin{tabular}{|l|l|l|}
\hline 1 & 1 & 3 \\
\hline 2 & 2 & \multicolumn{1}{|c}{} \\
\cline { 1 - 2 } &
\end{tabular}

\begin{tabular}{|l|l|l|}
\hline 1 & 1 & 2 \\
\hline 2 & 3 & \multicolumn{1}{|c}{} \\
\cline { 1 - 2 } &
\end{tabular}

Fig. 5. An example of two tableaux with content $(2,2,1)$ and shape $(3,2)$.

It is well known that the structure of a complex semisimple Lie algebra $\mathfrak{g}$ can be obtained from the discrete geometric information given by the root system [31]. Polytopes derived from the root system of $\mathfrak{g}$ and their lattice points appear in the study of a tensor product decomposition into irreducibles. If we consider a simple $\mathfrak{g}$-module $V_{\lambda}$ with highest weight $\lambda$ results of Berenstein and Zelevinsky [16] indicate that the multiplicity of the simple module $V_{\lambda}$ in the tensor product $V_{\mu} \otimes V_{\nu}$ for given dominant weights $\lambda, \mu, \nu$ is equal to the number of lattice points inside certain polytopes. Also related are the Kostant's partition function which appear in many classical formulas such as Steinberg's formula [31]. They can also be seen naturally as counting lattice points of certain polytopes.

- Another area of application is computational commutative algebra [48]. Given a finite set of monomials $m_{1}, m_{2}, \ldots, m_{k}$ in $\mathbb{C}\left[x_{1}, x_{2}, \ldots, x_{n}\right]$ one can consider the ring $\mathbb{C}\left[m_{1}, m_{2}, \ldots, m_{k}\right]$ generated by those monomials (i.e. like the usual polynomial ring but powers of the monomials $m_{i}$ replace powers of variables). Algebraists are interested on the Hilbert series of this ring with respect to various gradings (ways of measuring the degree of a monomial). The Hilbert series are generating functions for the vector space dimensions of the graded pieces of the ring. Barvinok and Woods [10] have recently shown that Hilbert series of a monomial algebra for the finest $\mathbb{Z}^{n}$ grading can be computed in polynomial time, via lattice point counting techniques, when the number of variables is fixed. Yet another connection to commutative algebra is the computation of Gröbner bases of toric ideals [22]. These, like usual vector space bases, allow many important calculations about the ideal.

\section{The Main Structure Theory}

By now I hope you are convinced of the interesting nature of the counting function $\phi_{A}(b)$, and maybe you wish to learn more about it. I will present what I would call the structure theory of these functions. The functions $\phi_{A}(b)$ are so basic and natural that one can easily believe their 
study goes back to Gauss, Euler or even earlier. Surprisingly the first direct investigations of $\phi_{A}(b)$ date back to the 1960's work of Eugène Ehrhart (1906-2000) [28,29] (with Pick's theorem as an sporadic earlier particular case). Ehrhart was a man with artistic and mathematical talents. As a lycée teacher he did many of his investigations as an amateur mathematician. To read more about him see the tribute written by Philippe Clauss http://icps.u-strasbg.fr/ ${ }^{\sim}$ clauss/Ehrhart.html.

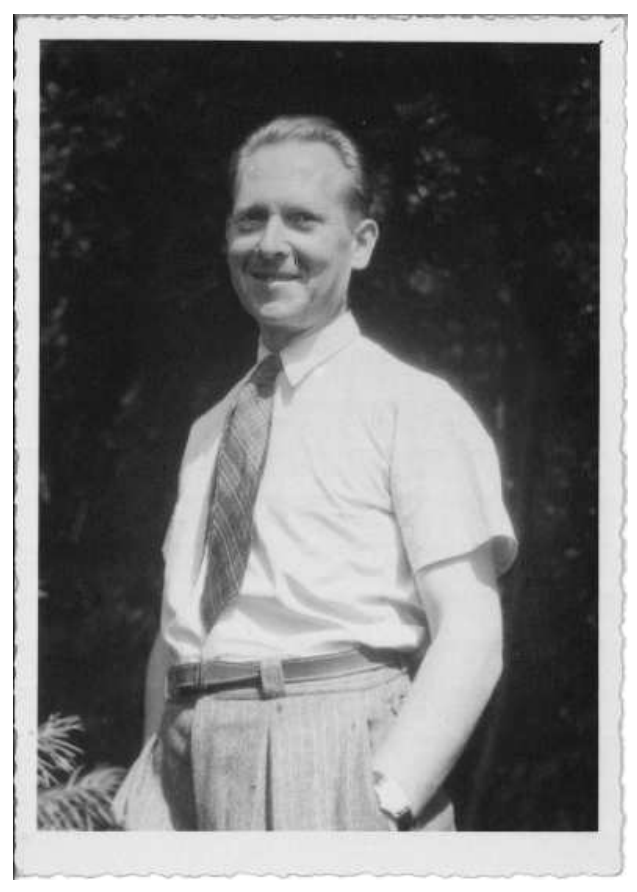

Fig. 6. Eugène Ehrhart (1906-2000).

Ehrhart began by considering the case when $b$ changes as a singleparameter dilation, i.e. $b=\lambda v$ for a fixed vector $v$. Geometrically this can be interpreted as inflating or dilating the associated polytope while leaving the angles and proportions fixed. More precisely, let $P$ be a convex polytope in $\mathbb{R}^{d}$. For each integer $n \geq 1$, define the dilation of $P$ by $n$ as the polyhedron $n P=\{n q: q \in P\}$ (see Figure 7). Thus for $P$ a $d$-polytope, Ehrhart studied the particular case of $\phi_{A}(b)$ given by the counting function $i(P, n)=\#\left(n P \cap \mathbb{Z}^{d}\right)=\#\left\{q \in P: n q \in \mathbb{Z}^{d}\right\}$. This is the number of lattice points in the dilation $n P$ of $P$. Similarly, if $P^{\circ}$ denotes the relative interior of $P$, i.e. $i\left(P^{\circ}, n\right)=\#\left\{q \in P-\partial P: n q \in \mathbb{Z}^{d}\right\}$. Let us take the simplest ever example, a unit square (see Figure 7 ). the reader can easily verify $i(P, n)=(n+1)^{2}$ and $i\left(P^{\circ}, n\right)=(n-1)^{2}$. In fact, for a $d$-dimensional unit cube we have $i(P, n)=(n+1)^{d}$. 


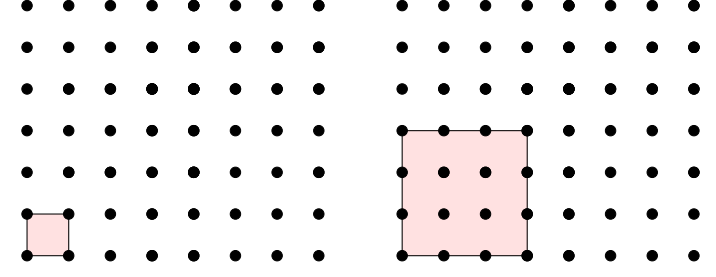

$\mathrm{P} \quad 3 \mathrm{P}$

Fig. 7. Dilating a unit square.

But if we just slightly translate the square to have non-integer coordinates, say vertices with coordinates $-1 / 2,1 / 2$, the task of computing $i(P, n)$ becomes more complicated. The correct description is given by the following theorem (see $[29,47,57]$ and the many references therein).

Theorem 1. Let $P$ be a convex d-dimensional rational polytope. Then for the univariate function $i(P, n)$ there exists an integer $N>0$ and polynomials $f_{0}, f_{1}, \ldots, f_{N-1}$ such that $i(P, n)=f_{i}(n)$ if $n \equiv i$ modulo $N$. We say that $i(P, n)$ is the Ehrhart quasipolynomial of $P$, in the dilation variable $n$. Moreover the non-zero polynomial components $f_{i}$ have degree $d=\operatorname{dim}(P)$ and their leading term equals the volume of $P$. When the coordinates of the vertices of $P$ are integers $i(P, n)$ is given by a single polynomial.

Why is the leading coefficient equal to the volume? Intuitively, dilating the polytope $P$ is equivalent to leaving $P$ as it is but refining the grid size of the lattice points. When we count lattice points we are essentially adding the volumes of tiny unit cubes centered at those lattice points which, as we refine the grid, approximate better and better the volume of the polytope: the main principle of the Riemann integral! A lot of the more recent work about Ehrhart counting functions was motivated by trying to understand the lower degree coefficients of the polynomial components. See for instance $[12,14,35,47,55]$. It is also important to mention that it can certainly happen that a polytope with non-integral coordinates has an Ehrhart polynomial $[49]$.

One possible generalization of Ehrhart's result is to count the lattice points with weights coming from a weight function $f$ instead of counting them as one, in other words one considers the function $i(P, f, n)=$ $\sum_{\alpha \in n P \cap \mathbb{Z}^{d}} f(\alpha)$. For example, if the polytope $P$ is the unit square $[0,1]^{2}$, and the weight function $f(x, y)$ is a polynomial of the form $x^{k} y^{k}$ with $0 \leq k \leq 3$, the corresponding weighted Ehrhart functions are 


$$
\begin{aligned}
i(P, n) & =n^{2}+2 n+1=(n+1)^{2}, \\
i(P, x y, n) & =\frac{1}{4} n^{4}+\frac{1}{2} n^{3}+\frac{1}{4} n^{2}, \\
i\left(P, x^{2} y^{2}, n\right) & =\frac{1}{9} n^{6}+\frac{1}{3} n^{5}+\frac{13}{36} n^{4}+\frac{1}{6} n^{3}+\frac{1}{36} n^{2}, \\
i\left(P, x^{3} y^{3}, n\right) & =\frac{1}{16} n^{8}+\frac{1}{4} n^{7}+\frac{3}{8} n^{6}+\frac{1}{4} n^{5}+\frac{1}{16} n^{4} .
\end{aligned}
$$

The degree of the polynomial $i\left(P, x^{k} y^{k}\right)$ is $2 k+2$, in general, it will be the dimension of the polytope plus the degree of the weight function. What is the leading coefficient now? Can you guess? See [19] for details.

Now we are interested on another generalization, when $\phi_{A}(b)$ is a multivariable function, $b$ can change differently in all entries. We present now a fairly general structure theorem (see $[15,58]$ ). To understand the statement the reader must think of cones. Given a finite set of vectors $S$, the cone cone $(S)$ is the collection of all nonnegative linear combinations of elements of $S$. We call the set $S$ the generators of the cone. Intuitively, cones look like ice cream cones, but with flat faces; see Figure 12 for a picture of a two-dimensional cone. When a cone does not contain a linear subspace we say the cone is pointed. In that case, we have a minimum set of generators, which we call the rays of the cone. As practice to familiarize yourself with cones, observe that the parameter space for $\phi_{A}(b)$ is cone $(A)$; only for $b \in \operatorname{cone}(A)$ the function $\phi_{A}(b)$ can be non-zero.

Theorem 2. For a $d \times n$ integral matrix $A$ and a parameter vector $b \in$ $\operatorname{cone}(A)$, there exist a finite decomposition of $\mathbb{Z}^{d} \cap \operatorname{cone}(A)$ such that $\phi_{A}$ is a multivariate polynomial of degree $n-d$ in each piece. The number $n-d$ is the dimension of the polytope $\{x: A x=b x \geq 0\}$.

More precisely, cone $(A)$ can be decomposed into convex polyhedral subcones of cone $(A)$, called chambers, such that, for all integral vectors $b$ inside a chamber the function $\phi_{A}(b)$ can be written as a fixed polynomial function of degree $n-d$ in the variables $b_{1}, \ldots, b_{d}$ plus a "correction polynomial" of smaller degree. The correction terms depend periodically on the values of $b_{1}, b_{2}, \ldots, b_{d}$.

Now we present two examples to illustrate Theorem 2. Let us begin with the $3 \times 6$ matrix 


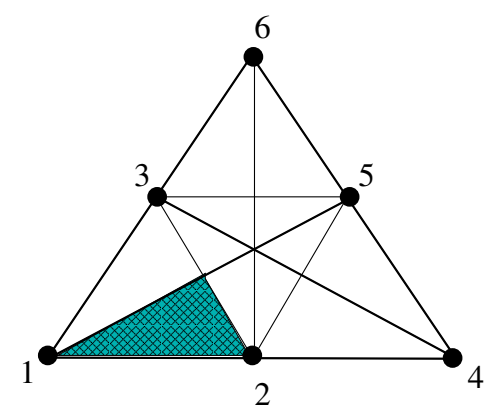

Fig. 8. A two-dimensional slice of cone $(A)$, example one.

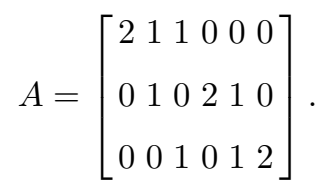

It is not a surprise that some "periodic" or "modular" components must appear in the final formulas for $\phi_{A}$. For example, observe that any vector $b=\left(b_{1}, b_{2}, b_{3}\right)$ which is a non-negative integer combination of the columns of $A$ must satisfy $b_{1}+b_{2}+b_{3} \equiv 0, \bmod 2$ because all columns of $A$ do. The cone cone $(A)$ is three dimensional, corresponding to the three entries of $b$. For the sake of being able to draw it, I showed only a 2-dimensional slice of the cone marking the 6 generating columns of $A$ with the numbers 1 to 6 . See Figure 8.

The theorem says there should be finitely many polynomials describing $\phi_{A}$. The cone is decomposed into 12 chambers, in this example the regions are triangular cones (in Figure 8 represented by triangles). Only 3 polynomials concern the formula of $\phi_{A}$ for those vectors inside the left-side chamber shaded in the picture; these are:

$$
\phi_{A}\left(b_{1}, b_{2}, b_{3}\right)=\frac{b_{2} b_{3}}{2}+\frac{b_{2} b_{3}^{2}}{8}-\frac{b_{3}^{2}}{24}+\text { correction }
$$

where

$$
\text { correction }=\left\{\begin{array}{lll}
1+\frac{b_{2}}{2}+\frac{2 b_{3}}{3} & \text { if } b_{1} \equiv 0 \text { and } b_{2} \equiv 0 & \bmod 2 \\
\frac{1}{2}+\frac{b_{2}}{2}+\frac{5 b_{3}}{12} & \text { if } b_{1} \equiv 1 \text { and } b_{2} \equiv 1 \\
\frac{1}{2}+\frac{3 b_{2}}{8}+\frac{13 b_{3}}{24} & \text { otherwise }
\end{array}\right.
$$



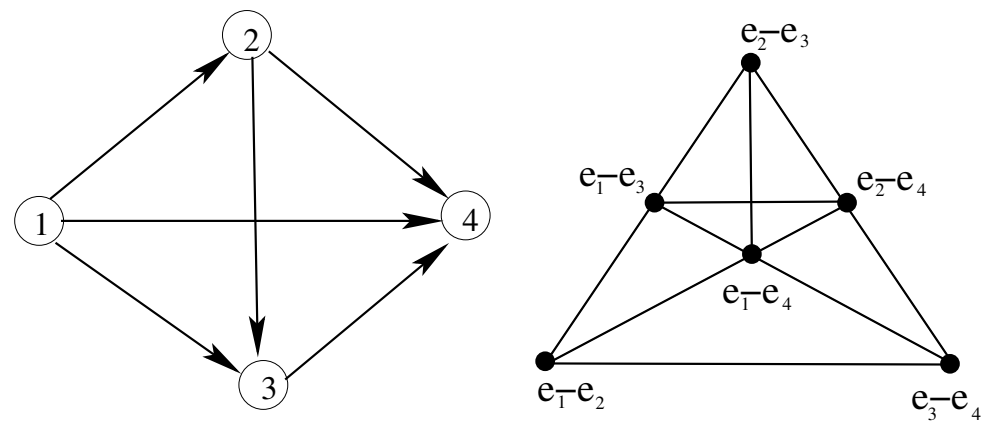

Fig. 9. The digraph that gives matrix $A$ for example two and the seven chambers for $\phi_{A}$ (a two-dimensional representation).

Figure 9 shows a second example where the matrix $A$ is very special. $A$ is defined to be the node-arc incidence matrix of the digraph on the left of the figure. Thus $A$ is given by the pairwise differences of standard unit vectors $e_{i}$. For instance the last column of $A$ is just $e_{3}-e_{4}$ (in this way we marked the six columns of the matrix in Figure 9). Remarkably, in this example there is a single polynomial (no correction terms) in each chamber. Therefore seven polynomials define the whole counting function $\phi_{A}$, one per triangular cone in the figure (there only represented by a triangle). The reason for this is that the matrix $A$ is totally unimodular [53]. In particular, the vertices of the polytopes $\{x: A x=b, x \geq 0\}$ are integral for all integral $b$. If you recall Theorem 1 , you can see $\phi_{A}(t b)$ is actually a polynomial (not a quasipolynomial) for any fixed $b$ in the variable $t$. Of the seven polynomials, take as an example the one for the biggest triangular cone region, with corners $e_{1}-e_{2}, e_{1}-e_{4}$, and $e_{3}-e_{4}$, it is

$$
\phi_{A}(b)=\left(b_{1}+b_{2}+3\right)\left(b_{1}+b_{2}+2\right)\left(b_{1}+b_{2}+1\right) / 6 .
$$

This is valid for all vectors integral $b$ within this triangular cone.

What are these mysterious chambers? Their structure is rather pretty and worth studying further. Let $\Delta$ be the set of column vectors of the input matrix $A$ (defining our polytope), this means we consider the column vectors of the matrix $A$ without multiplicities. The chamber complex is a special polyhedral subdivision of the cone generated by the nonnegative linear combinations of elements in $\Delta$, denoted by $C(\Delta)$. It is defined as the common refinement of the simplicial cones $C(\sigma)$ running over all possible subsets of $\Delta$ which form a basis for $\mathbb{R}^{d}$. More precisely, denote by $\Sigma_{\Delta}$ the set of all simplicial cones whose extreme rays, are generated by elements of $\Delta$, If $\sigma$ is an element of $\Sigma_{\Delta}$ and $\partial \sigma$ denotes its boundary and $\partial \Sigma_{\Delta}$ the union of the boundaries of all simplicial cones, then the complement of $\Sigma_{\Delta}$ inside $C(\Delta)$ consists of the disjoint union of open convex cells. See Figure 
10 for a two-dimensional slice view of a chamber complex of a pentagonal cone. The refinement we described gives eleven chambers or regions. One can think of them as the result of overlapping all the possible triangulations of the cone. One important fact is that finding the chamber that contains a particular vector $b$ is equivalent to enumerating the vertices of the polytope $\{x: A x=b, x \geq 0\}$.

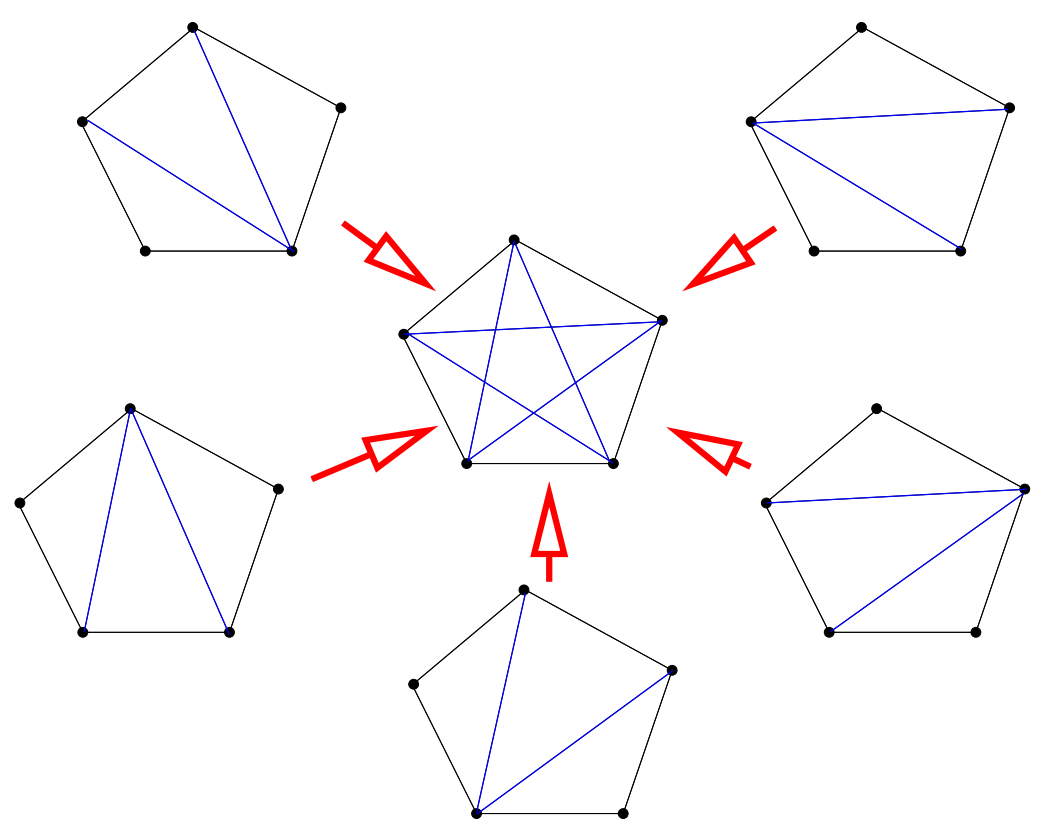

Fig. 10. The construction of the chamber complex of a pentagonal cone (shown as a 2-dimensional slice).

\section{Actually Counting: Modern Algorithms and Software}

Counting lattice points is difficult: We know that when the dimension is an input variable the problem of detecting a lattice point in polyhedra is $N P$-hard [32]. Already counting $2 \times n$ contingency tables is \#P-hard [27]. Branch-and-Bound techniques and exhaustive enumeration work to some extent, but the method gets stuck in surprisingly small problems, e.g. hard knapsack problems. Exhaustive enumeration techniques are not useful to derive formulas as they depend on the size of the right-hand-side vector $b$.

During the 1980's and 1990's a new breed of algorithms and counting formulas were created. They rely on deeper algebraic and analytic properties 
of polyhedra. Some authors emphasize the more algebraic-geometric nature of the lattice points of polyhedra via the theory of toric varieties, while others make a much stronger use of complex analysis and convexity (see [9, $6,26,40,43,50,54,52]$ and the many references within).

Several researchers have even produced software for counting lattice points in polyhedra. For instance, Lisonek created a Maple package that works on knapsack type problems [44]. The Beck-Pixton software [13] can perform fast computations with two-dimensional transportation polytopes. They have the best computation times for that family of polytopes. Vergne and collaborators (see [6]) have developed a package special for unimodular matrices which is very fast. Working with general polytopes we have two programs: one is ehrhart, developed by Clauss, Loechner and Wilde (http://icps.u-strasbg.fr/Ehrhart/program/). The first implementation of Barvinok's algorithm was the software LattE developed at UC Davis $[23,24]$. LattE counts lattice points, generates Ehrhart's quasipolynomials, and even solves small integer programs. Now we wish to sketch some of the ideas involved in some the algorithms. As the reader will see we have a bias for the use of rational functions. We sketch Barvinok's algorithm first, then we sketch and refer to other algorithms that use again rational functions. As of today, these rational-function-based algorithms are the fastest available.

\subsection{Barvinok's algorithm}

In 1993 A. Barvinok [8,9] found an algorithm to count integer points inside polyhedra. When the dimension is fixed the algorithm can count the number of lattice points in a polytope in polynomial time on the size of the input (the size is given by the binary encoding of the data). The key ideas are using rational functions as efficient data structures and the unimodular signed decomposition of polyhedra. Given a convex polyhedron $P$ (not necessarily a polytope anymore!), we wish to compute the multivariate generating function

$$
f(P ; x)=\sum_{\alpha \in P \cap \mathbb{Z}^{d}} x^{\alpha},
$$

where $x^{\alpha}=x_{1}^{\alpha_{1}} x_{2}^{\alpha_{2}} \ldots x_{d}^{\alpha_{d}}$. This is an infinite formal power series if $P$ is not bounded, but if $P$ is a polytope it is a (Laurent) polynomial with one monomial per lattice point. For example, if we consider a rectangle with vertices $V_{1}=(0,0), V_{2}=(5000,0), V_{3}=(0,5000)$, and $V_{4}=(5000,5000)$ the generating function $f(P)$ has over 25,000,000 monomials,

$$
f\left(P, z_{1}, z_{2}\right)=1+z_{1}+z_{2}+z_{1} z_{2}^{2}+z_{1}^{2} z_{2}+\cdots+z_{1}^{5000} z_{2}^{5000} .
$$

The representation of $f\left(P ; z_{1}, z_{2}\right)$ as monomials is clearly way too long to be of practical use. But Barvinok's method instead rewrites it as a compact 
sum of rational functions. For instance, only four rational functions suffice to represent the over 25 million monomials. Indeed $f\left(P, z_{1}, z_{2}\right)$ equals

$\frac{1}{\left(1-z_{1}\right)\left(1-z_{2}\right)}+\frac{z_{1}^{5000}}{\left(1-z_{1}^{-1}\right)\left(1-z_{2}\right)}+\frac{z_{2}{ }^{5000}}{\left(1-z_{2}^{-1}\right)\left(1-z_{1}\right)}+\frac{z_{1}{ }^{5000} z_{2}{ }^{5000}}{\left(1-z_{1}{ }^{-1}\right)\left(1-z_{2}{ }^{-1}\right)}$

Note that if we wish to know the number of lattice points in $P$, and we knew $f(P ; z)$, we could compute it as the limit when the vector $\left(x_{1}, \ldots, x_{n}\right)$ goes to $(1,1, \ldots, 1)$. Similarly the maximum of a linear functional over the lattice points equals the highest degree of the univariate polynomial one gets after doing a monomial substitution $x_{i} \rightarrow t^{c_{i}}$ (See [10]). These two calculations can be difficult because to the poles of the rational functions. One uses complex analysis (residue calculations) to find the answer.

A beautiful theorem of M. Brion [18] says that to compute the rational function representation of $f(P ; z)$ it is enough to do it for tangent cones at each vertex of $P$. Let $P$ be a convex polytope and let $V(P)$ be the vertex set of $P$. Let $K_{v}$ be the tangent cone at $v \in V(P)$. This is the (possibly translated) cone defined by the facets touching vertex $v$ (see Figure 11). Then the following nice formula holds:

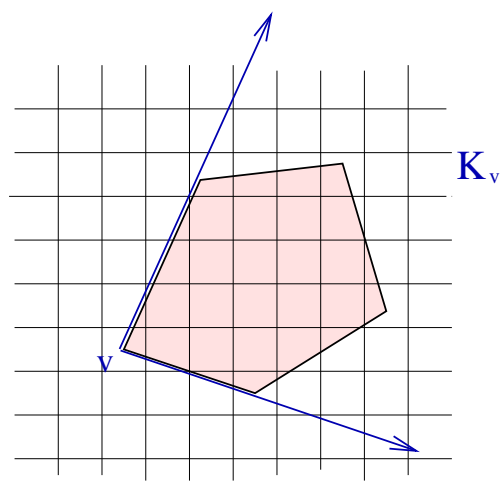

Fig. 11. The tangent cone at vertex $v$

$$
f(P ; z)=\sum_{v \in V(P)} f\left(K_{v} ; z\right) .
$$

Since it is enough to compute everything for cones, we first explain how to compute the rational function for the "smallest" cones, simple cones. A cone is said to be simple if its rays are linearly independent vectors. For 


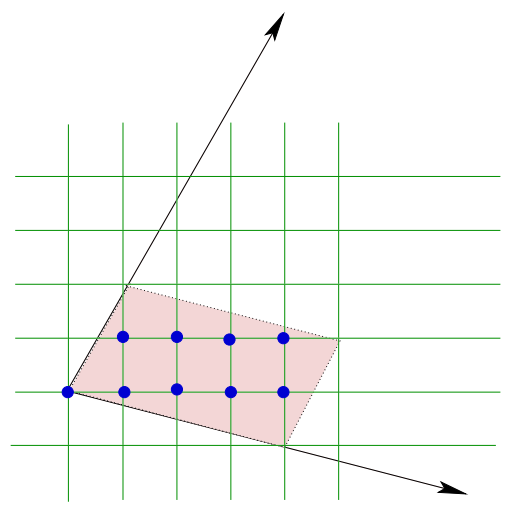

Fig. 12. A two dimensional cone (its vertex at the origin) and its fundamental parallelepiped

instance all the tangent cones of the pentagon of Figure 11 are simple. Obtaining the rational function representation of the lattice points of a simple cone $K \subset \mathbb{R}^{d}$, is easy (see all details in [55] or in Chapter IV of [57]). Here is the formula, that one can write directly from the coordinates of the rays of the cone and its fundamental parallelepiped $\Pi$ :

$$
f(K ; z)=\frac{\sum_{u \in \Pi \cap \mathbb{Z}^{d}} z^{u}}{\left(1-z^{c_{1}}\right)\left(1-z^{c_{2}}\right) \ldots\left(1-z^{c_{d}}\right)} .
$$

Here $\Pi$ is the half open parallelepiped $\left\{x: x=\alpha_{1} c_{1}+\cdots+\alpha_{d} c_{d}, 0 \leq\right.$ $\left.\alpha_{i}<1\right\}$. We can do a two-dimensional example shown in Figure 12: we have $d=2$ and $c_{1}=(1,2), c_{2}=(4,-1)$. We have:

$$
f(K ; z)=\frac{z_{1}^{4} z_{2}+z_{1}^{3} z_{2}+z_{1}^{2} z_{2}+z_{1} z_{2}+z_{1}^{4}+z_{1}^{3}+z_{1}^{2}+z_{1}+1}{\left(1-z_{1} z_{2}^{2}\right)\left(1-z_{1}^{4} z_{2}^{-1}\right)} .
$$

But what to do if the cone $K$ is not simple? Break it into simple cones! The wonderful idea of A. Barvinok was noting that, although triangulating the cone $K$ may be an inefficient way to subdivide the cone (i.e. exponentially many pieces), if one is willing to add and substract cones for fix dimension $d$, there exists a polynomial time algorithm which decomposes a rational polyhedral cone $K \subset \mathbb{R}^{d}$ into simple unimodular cones $K_{i}$. A simple cone is unimodular if its fundamental parallelepiped there is a single lattice point. See Figure 13. In fact, via the decomposition, with numbers $\epsilon_{i} \in\{-1,1\}$ we can write an expression 


$$
f(K ; z)=\sum_{i \in I} \epsilon_{i} f\left(K_{i} ; z\right), \quad|I|<\infty
$$

The index set $I$ is of size polynomial in the input data as long as we work in fixed dimension.
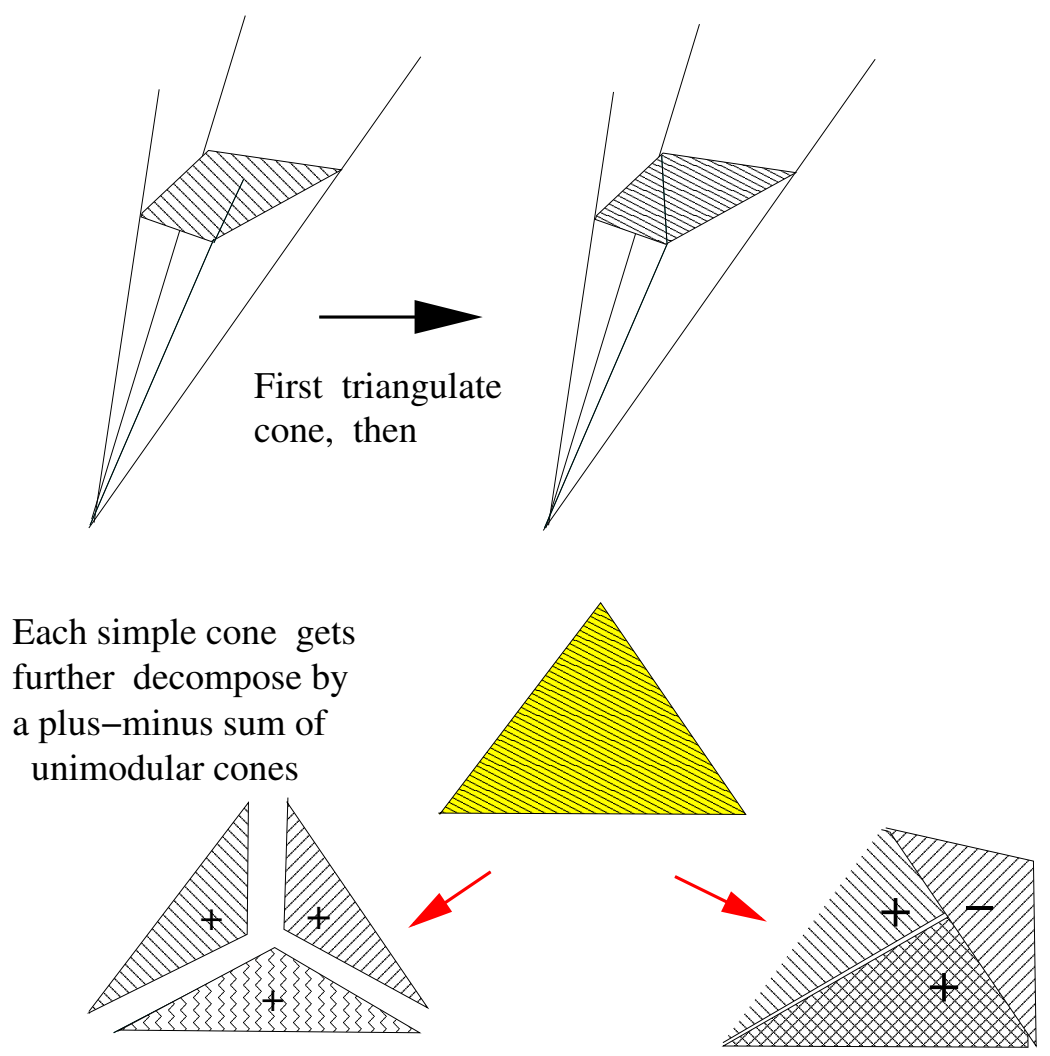

Fig. 13. The signed decomposition of a cone into unimodular cones. Two general steps

\subsection{More methods using rational functions}

Recall $\phi_{A}(b)=\#\{x: A x=b, x \geq 0$, integer $\}$. If we denote by $A_{i}$ the columns of the matrix $A$, then it is easy to see that 


$$
\sum \phi_{A}(b) z^{b}=\frac{1}{\prod_{j=1}^{n}\left(1-z^{A_{j}}\right)} .
$$

It is also fairly easy to see that:

$$
\sum_{b \in \operatorname{cone}(A) \cap \mathbb{Z}^{n}} \phi_{A}(b) e^{-\langle b, z\rangle}=\frac{1}{\prod_{A_{i} \in A} 1-e^{-\left\langle A_{i}, z\right\rangle}} .
$$

How to invert this equations to recover $\phi_{A}$ ? Some authors have taken a complex analytic view. For instance, in [13] the integral is evaluated by repeated application of Cauchy's integral theorem and clever manipulations.

$$
\phi_{A}(b)=\frac{1}{(2 \pi i)^{m}} \int_{\left|z_{1}\right|=\epsilon_{1}} \cdots \int_{\left|z_{m}\right|=\epsilon_{m}} \frac{z_{1}^{-b_{1}-1} \cdots z_{m}^{-b_{m}-1}}{\left(1-z^{A_{1}}\right) \cdots\left(1-z^{A_{d}}\right)} d z
$$

Here $0<\epsilon_{1}, \ldots, \epsilon_{m}<1$ are different numbers such that we can expand all the $\frac{1}{1-z^{M_{k}}}$ into the power series about 0 . This method has been applied very successfully to determine the volumes of Birkhoff polytopes in [13]. The reader may recall from basic calculus that to integrate rational functions it was sometimes useful to use a partial fraction decomposition, in order to simplify the expressions. M. Vergne and collaborators have exploited the structure of the hyperplane arrangement associated to the columns of the matrix $A$, namely

$$
H_{A}=\bigcup_{A_{i} \text { column of } A}\left\{z \in \mathbb{C}^{r} \mid\left\langle A_{i}, z\right\rangle=0\right\}
$$

This was used to do a nice "simpler fraction" decomposition of the formula $\frac{1}{\prod_{j=1}^{n}\left(1-z^{A_{j}}\right)}$. A subset $\sigma$ of columns of $A$ is called basic if the elements $A_{i} \in \sigma$ form a vector space basis for $\mathbb{R}^{d}$. For such $\sigma$, set

$$
f_{\sigma}(z):=\frac{1}{\prod_{A_{i} \in \sigma}\left\langle A_{i}, z\right\rangle}
$$

We consider the vector space $S_{A}$ the spanned by such "simple" elements $f_{\sigma}$. Brion and Vergne [19] proved that there is a direct sum decomposition of the space $R_{A}$ of rational functions whose poles are on the hyperplane 
arrangement $H_{A}$ and $S_{A}$ is one of the summands. The projection map $T R$ : $R_{A} \longrightarrow S_{A}$ is called the total residue map. This projection allows to survive only simple summands that come from special bases. See $[6,19,54]$ and references within for more details and implementation.

\section{Beyond Polytopes}

To conclude this survey we would like to comment on what happens when we try to extend counting lattice points inside other region of space besides polytopes. We will see very interesting effects. Consider first the region $K(n)=\left\{(x, y): 1 \leq x \leq n, \frac{n}{x} \leq y \leq n\right\}$ bounded by a hyperbola and lines. Let $L$ be the open strip properly in between the hyperbolas $y=\frac{n}{x}$ and $y=\frac{n+1}{x}$, as denoted in Figure 14. Thus $L$ does not include the boundary of $K(n)$ nor that of $K(n+1)$. See Figure 14 .

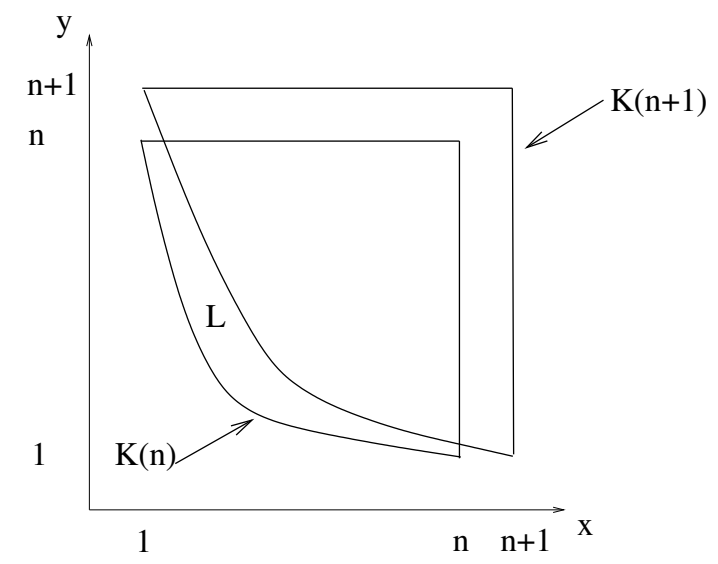

Fig. 14. The regions $K(n), K(n+1)$, and $L$.

Given all of this, we arrive at the following proposition:

Proposition 1. The number of lattice points inside the planar non-convex region

$$
R=(K(n+1) \backslash K(n)) \cup(K(n) \backslash K(n+1))
$$

equals $2 n+3$ if and only if $n$ is prime.

Here is why: observe that if $n$ is not a prime, then there is at least one $(a, b)$ such that $(a, b)=n$ and $(a, b)$ is on the lower boundary of $K(n)$ but $(a, b) \neq(n, 1)$ or $(1, n) . L$ has no lattice points otherwise one such $(c, d)$ will 
have $\frac{n+1}{c}>d>\frac{n}{c}$, however, this implies that $n<c d<n+1$ which is a contradiction since no integer can exist between consecutive integers. Thus the lattice points in the region $R$ are the $2 n+1$ lattice points along the lines $x=n+1$ and $y=n+1$, plus at least four more $(a, b),(b, a),(n, 1),(1, n)$ along the lower hyperbola. Next suppose $n$ is prime. Again, as we argued before, we have that the region $L$ does not contribute any lattice points. The lattice points in the region $R$ are the $2 n+1$ points along the lines $x=n+1$ and $y=n+1$, plus exactly two more $(n, 1),(1, n)$.

Already a simple variation of the construction with the region $K(a, b)=$ $\{(x, y): 1 \leq x \leq a, 1 \leq y \leq b, x y \geq b\}$ allows to count the number of integer divisors of $b$ in the interval $[1, a]$. When the sets for which we count lattice points are arbitrary the problem gets harder and harder as the dimension of the set grows: Given $(a, b, c)$ positive integers, deciding whether there is a lattice point in the set $\left\{x: a x^{2}+b x=c, x \geq 0\right\}$ is an NP-complete problem [2]. Finally, deciding whether there is a non-negative integer root for arbitrary polynomials in $\mathbb{Z}\left[x_{1}, \ldots, x_{9}\right]$ is undecidable, i.e. roughly speaking there is no computer algorithm to solve this problem for all instances [39]. Thus we clearly need to be less ambitious!

A convex set $C$ is a set of Euclidean space such that for any pair of points in $C$ the line segment joining $x$ and $y$ is completely inside $C$. Polyhedra are the simplest convex sets in a way. Thus it is natural to ask whether other convex sets besides polyhedra allow a nice theory of counting lattice points like the one we outlined in previous sections. Alas, this still contains some interesting surprises! In a way counting points inside general convex sets is an older problem. Already Gauss proposed a famous counting problems for circles [34]:
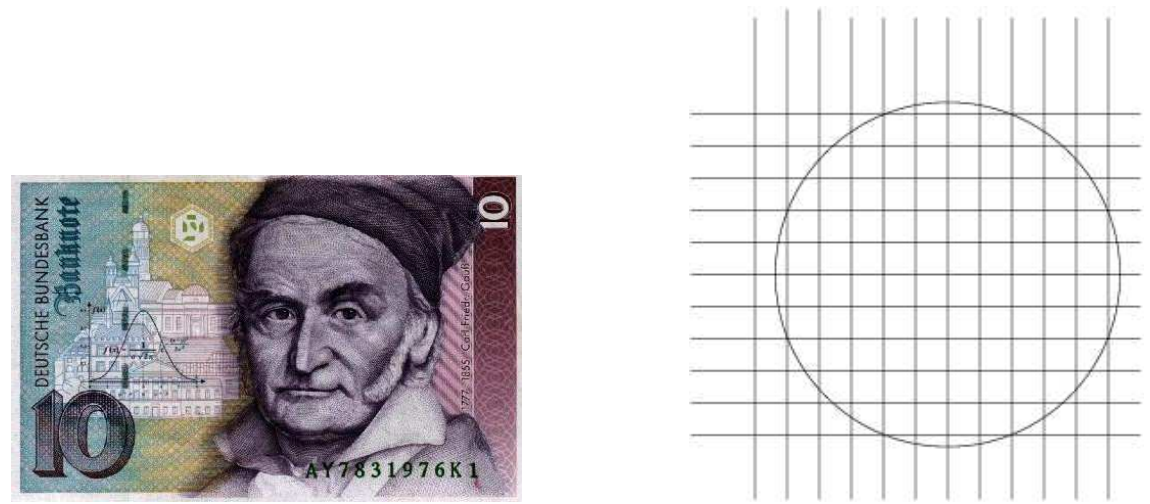

Fig. 15. Gauss and the circle area approximation problem

Suppose you are given a circle of radius $r$ centered at the origin. How good is the approximation of the number of lattice points $N(r)$ to the area 
of the circle as $r$ grows? In other words, what is the exact order of magnitude for the error function $N(r)-\pi r^{2}$ ? The answer is still not known exactly, although it is supposed to be in between $r^{.5}$ and $r^{.6301369863}$ (see [36]).

Counting lattice points in (four dimensional) convex bodies is something that credit card cyber-thieves would care about too! The reason is the RSA encryption algorithm relies on the difficulty of factorizing a number of the form $p q$ with $p, q$ large primes. Here is a way to factorize via counting lattice points: For an integer number $n=p q$ consider the 4-dimensional ball $B(n)=\left\{x \in R^{4}: x_{1}^{2}+x_{2}^{2}+x_{3}^{2}+x_{4}^{2} \leq n\right\}$. Jacobi (see $[4,37]$ ) proved that the number of ways in which a positive integer can be written as a sum of four squares is eight times the sum of its divisors that are not a multiple of four. So, for example there are 744 ways of writing 100 as sum of four squares because $1,2,5,10,25,50$ are the only divisors of 100 not divisible by 4. Thus if we know that $n=p q$, and $|B(n)|$ denotes the number of lattice points in $B(n)$ we have $|B(n)|-|B(n-1)|=8(1+p+q+n)$. Therefore a factorization of $n$ could be done fast if we knew how to compute $|B(n)|$ fast. Thus the RSA cryptosystems used in internet transactions could be broken if you knew how to quickly count lattice points inside four dimensional balls. This is a very intriguing connection.

Acknowledgements: I thank the anonymous referees for the many corrections. I am grateful to Günter M. Ziegler and Jürgen Wolfart for their help and suggestions for improvement. I thank Philippe Clauss who kindly provided references and the photograph we used of E. Ehrhart.

\section{References}

1. Aardal, K., Lenstra, A.K., and Lenstra, H.W. Jr.: Hard equality constrained integer knapsacks. In: W.J. Cook and A.S. Schulz (eds.), Integer Programming and Combinatorial Optimization: 9th International IPCO Conference, Lecture Notes in Computer Science vol. 2337, Springer-Verlag, 350-366, 2002.

2. Adleman, L. and Manders, K.: NP-complete decision problems for quadratic polynomials. In: Proceedings of the Eighth annual ACM symposium on theory of computing, Hershey PA 1976, 23-29, ACM, New York, 1976.

3. Ahmed, M., De Loera, J., and Hemmecke, R.: Polyhedral cones of magic cubes and square. In: New Directions in Combinatorial Geometry, The GoodmanPollack festschrift (eds. Aronov et al.), Springer Verlag, 25-41, 2003.

4. Andrews, G., Ekhad, S., and Zeilberger, D.: A short proof of Jacobi's formula for the number of representations of an integer as a sum of four squares, American Math. Monthly, 100, 274-276, 1993.

5. Balcioglu, A. and Wood R.: Enumerating Near-Min s-t Cuts. In: D.L. Woodruff (editor) Network Interdiction and Stochastic Integer Programming, vol. 22, series operations research/computer science interfaces, Kluwer, Boston 2002. 
6. Baldoni-Silva, W., De Loera, J., and Vergne, M.: Counting integral flows on Networks, Foundations of Computational Mathematics, Vol. 4, 277-314, 2004.

7. Barvinok, A.I.: A course in Convexity, Graduate studies in Mathematics, vol. 54, Amer. Math. Soc., Providence RI, 2002.

8. Barvinok, A.I.: Polynomial time algorithm for counting integral points in polyhedra when the dimension is fixed, Math of Operations Research, Vol. 19, 769-779, 1994.

9. Barvinok, A.I. and Pommersheim, J.: An algorithmic theory of lattice points in polyhedra. In: New Perspectives in Algebraic Combinatorics (Berkeley, CA, 1996-1997), 91-147, Math. Sci. Res. Inst. Publ. 38, Cambridge Univ. Press, Cambridge, 1999.

10. Barvinok, A.I. and Woods, K.: Short rational generating functions for lattice point problems, J. Amer. Math. Soc., Vol. 16, 957-979, 2003.

11. Beck, M.: Counting lattice points by means of the residue theorem. Ramanujan Journal, 4, no. 3, 299-310, 2000.

12. Beck, M., De Loera, J.A., Develin, M., Pfeifle, J., and Stanley, R.: Coefficients and roots of Ehrhart polynomials to appear in Cont. Math. (Proceedings of the Summer Research Conference on Integer Points in Polyhedra, July 13 July 17, Snowbird Utah, 2003).

13. Beck, M. and Pixton, D.: The Ehrhart polynomial of the Birkhoff polytope. Discrete Comp. Geom, 30, no. 4, 623-637, 2003.

14. Betke, U. and McMullen, P.: Lattice points in lattice polytopes, Monatsh. Math, 99, 253-265, 1985.

15. Blakley, G.R.: Combinatorial remarks on partitions of a multipartite number, Duke Math. J., 31, 335-340, 1964.

16. Berenstein, A. and Zelevinsky, A.: Tensor product multiplicities, canonical bases and totally positive varieties, Invent. Math. 143, 77-128, 2001.

17. Billey S., Guillemin V., and Rassart E.: A vector partition function for the multiplicities of $\mathfrak{s l}_{k} \mathbb{C}$, Journal of Algebra, vol. 278, 251-293, 2004.

18. Brion, M.: Points entiers dans les polyèdres convexes. Ann. Sci. École Norm. Sup., Vol. 21, 653-663, 1988.

19. Brion, M. and Vergne, M.: Residue formulae, vector partition functions and lattice points in rational polytopes, J. Amer. Math. Soc., Vol. 10, 797-833, 1997.

20. Clauss, P. and Loechner, V.: Parametric Analysis of Polyhedral Iteration Spaces, Journal of VLSI Signal Processing, Vol. 19, 179-194, 1998.

21. De Loera, J.A. and McAllister, T.B.: Vertices of Gelfand-Tsetlin polytopes Discrete Comp. Geom., vol 32, 459-470, 2004.

22. De Loera, J.A., Haws, D., Hemmecke, R., Huggins, P., Sturmfels B., and Yoshida R.: Short rational functions for toric algebra and applications J. Symbolic Comp., vol 38, 959-973, 2004.

23. De Loera, J. A., Haws, D., Hemmecke, R., Huggins, P., Tauzer, J. and Yoshida, R.: A User's Guide for LattE, software package LattE and manual are available at http://www.math.ucdavis.edu/ latte/ 2003.

24. De Loera, J. A. and Hemmecke, R. and Tauzer, J. and Yoshida, R.: Effective lattice point counting in rational convex polytopes, J. Symbolic Comp., vol. 38, 1273-1302, 2004.

25. Diaconis, P. and Gangolli, A.: Rectangular arrays with fixed margins, In: Discrete probability and algorithms (Minneapolis, MN, 1993), 15-41, IMA Vol. Math. Appl., 72, Springer, New York, 1995. 
26. Diaz, R. and Robins, S.: The Ehrhart Polynomial of a Lattice Polytope, Annals of Mathematics, 145, 503-518, 1997.

27. Dyer, M., Mount, D., Kannan, R. and Mount, J.: Sampling contingency tables, Random Structures and Algorithms, Vol. 10, 487-506, 1997.

28. Ehrhart, E.: Sur un problème de géométrie diophantienne linéaire. I. Polyèdres et réseaux,, J. Reine Angew. Math., Vol. 226, 1-29, 1967.

29. Ehrhart, E.: Polynômes arithmétiques et méthode des polyèdres en combinatoire, International Series of Numerical Mathematics, Vol. 35, Birkhäuser Verlag, Basel, 1977.

30. Fienberg, S.E. and Makov, U.E. and Meyer, M.M. and Steele, R.J.: Computing the exact conditional distribution for a multi-way contingency table conditional on its marginal totals. In: Data Analysis From Statistical Foundations, 145-166, Nova Science Publishers, A. K. Md. E. Saleh, Huntington, NY, 2001.

31. Fulton, W. and Harris, J.: Representation Theory: A first course, Graduate texts in Mathematics, vol 129, Springer, New York, 1991.

32. Garey, M.R. and Johnson, S.J.: Computers and Intractability: A Guide to the Theory of NP-Completeness, Freeman, San Francisco, 1979.

33. Ghosh, S., Martonosi, M., Malik, S.: Cache miss equations: a compiler framework for analyzing and tuning memory behavior, ACM transactions on programming languages and systems, 21, 703-746, 1999.

34. Guy, R. K.: Unsolved Problems in Number Theory, 2nd ed. New York: Springer-Verlag, 240-241, 1994.

35. Hibi, T.: A lower bound theorem for Ehrhart polynomials of convex polytopes, Advances in Math. 105, 162-165, 1994.

36. Huxley, M.N.: Area, lattice points, and exponential sums, Oxford Univ. Press. Oxford, 1996.

37. Jacobi, C.: Gesammelte Werke, Berlin 1881-1891. Reprinted by Chelsea, New York, 1969.

38. Jerrum, M. and Sinclair, A.: The Markov Chain Monte Carlo Method: An approach to approximate Counting and integration, in: Dorit Hochbaum (Ed.), Approximation Algorithms, PWS Publishing Company, Boston, 1997.

39. Jones, J.P.: Universal diophantine equations, Journal of symbolic logic, 47 (3), 403-410, 1982.

40. Kantor, J.M. and Khovanskii, A.: Une application du Théorème de RiemannRoch combinatoire au polynôme d'Ehrhart des polytopes entier, C. R. Acad. Sci. Paris, Series I, Vol. 317, 501-507, 1993.

41. Knutson, A. and Tao, T.: The honeycomb model of $G L_{n}(\mathbb{C})$ tensor products I: Proof of the Saturation Conjecture, J. Amer. Math. Soc. 12, 1055-1090, 1999.

42. Knutson, A., Tao, T., and Woodward, C.: The honeycomb model of $G L_{n}(\mathbb{C})$ tensor products II: Puzzles determine facets of the Littlewood-Richardson cone, J. Amer. Math. Soc, 17, 19-48, 2004.

43. Lasserre, J.B. and Zeron, E.S.: On counting integral points in a convex rational polytope Math. Oper. Res. 28, 853-870, 2003.

44. Lisonek, P.: Denumerants and their approximations, Journal of Combinatorial Mathematics and Combinatorial Computing, Vol. 18, 225-232, 1995.

45. Loechner, V., Meister, B., and Clauss, P.: Precise data locality optimization of nested loops, J. Supercomput., Vol. 21, 37-76, 2002.

46. Lóvász, L. and Plummer, M.: Matching Theory North-Holland mathematics studies, Elsevier, 1986. 
47. Macdonald, I. G.: Polynomials associated with finite cell-complexes, J. London Math. Soc. (2), 4, 181-192, 1971.

48. Miller, E. and Sturmfels, B.: Combinatorial Commutative Algebra, Graduate texts in Mathematics, Vol. 227, Springer, New York, 2004.

49. McAllister, T. B. and Woods, K.: The minimum period of the Ehrhart quasipolynomial of a rational polytope, Journal of Combinatorial Theory, Series A, to appear.

50. Morelli, R.: Pick's theorem and the Todd class of a toric variety, Adv. Math. 100, 183-231, 1993.

51. Moura, L.: Polyhedral methods in design theory, in Computational and Constructive Design Theory, 227-254, W.D. Wallis (ed.), Math. Appl. 368, Kluwer, 1996.

52. Pemantle, R. and Wilson, M.: Asymptotics of multivariate sequences, part I: smooth points of the singular variety. J. Comb. Theory, Series A, vol. 97, 129-161, 2001.

53. Schrijver, A.: Theory of Linear and Integer Programming. Wiley-Interscience, 1986.

54. Szenes A. and Vergne M.: Residue formulae for vector partitions and EulerMaclaurin sums, Adv. in Appl. Math, 30, 295-342, 2003.

55. Stanley, R. P.: Decompositions of rational convex polytopes. In: Combinatorial mathematics, optimal designs and their applications (Proc. Sympos. Combin. Math. and Optimal Design, Colorado State Univ., Fort Collins, Colo., 1978), Ann. Discrete Math.,6, 333-342, 1980.

56. Stanley. R.P.: Two poset polytopes, Discrete Computational Geometry, Vol. 1, 9-23, 1986.

57. Stanley, R.P.: Enumerative Combinatorics,Volume I, Cambridge, 1997.

58. Sturmfels, B.: On Vector Partition functions, J. Combin. Theory Ser. A, Vol. 72, 302-309, 1995.

59. Welsh, D.J.A.: Approximate counting in Surveys in Combinatorics, (edited by R. A. Bailey), Cambridge Univ. Press, Cambridge, 1997.

60. Ziegler, G.M.: Lectures on Polytopes, Springer-Verlag, New York, 1995. 